\title{
Clinical Importance of PRAME Gene Expression in Acute Myeloid Leukemia
}

\author{
Ahmad Baraka $^{1 *}$, Mona E. Hashem ${ }^{1}$, Shereen ElShorbagy ${ }^{2}$, Mona hassanein ${ }^{2}$ and Salah F. Elsayed ${ }^{3}$ \\ ${ }^{1}$ Department of Clinical Pathology, Faculty of Medicine, Zagazig University, Zagazig, Egypt \\ ${ }^{2}$ Department of Medical Oncology, Faculty of Medicine, Zagazig University, Zagazig, Egypt \\ ${ }^{3}$ Department of Internal Medicine, Faculty of Medicine, Zagazig University, Zagazig, Egypt \\ *Corresponding author: Baraka A, Clinical Pathology department, Faculty of Medicine, Zagazig University, 44519, Zagazig, Egypt, Tel: 01005648997; E-mail: \\ barakalab@yahoo.com
}

Rec date: Oct 14, 2016; Acc date: Nov 17, 2016; Pub date: Nov 21, 2016

Copyright: (c) 2016 Baraka A, et al. This is an open-access article distributed under the terms of the Creative Commons Attribution License, which permits unrestricted use, distribution, and reproduction in any medium, provided the original author and source are credited.

\begin{abstract}
Background: The PRAME (preferentially expressed antigen of melanoma) gene is frequently over expressed in a wide variety of malignant diseases, including acute myeloid leukemia (AML).

Objective:To investigate the expression of PRAME gene in AML and its importance in the prognosis.

Patients and methods The transcript level of PRAME gene was detected in peripheral blood specimens from 72 de novo AML patients and 40 control subjects by using real-time quantitative PCR and the prognostic value of PRAME was determined through treatment outcome and survival analysis.

Results:PRAME gene was highly expressed in $74 \%$ of de novo AML cases while low expression was detected among healthy controls, the mean value of PRAME gene expression was highest among M3 AML subtype and lowest among M5b AML subtype. No significant correlation could be observed between PRAME gene expression and different $\mathrm{CBC}$ parameters, age, gender or cytogenetics, but there was a significance positive correlation between PRAME gene expression with treatment outcome, disease free survival (DFS) and overall survival.
\end{abstract}

Conclusion:PRAME gene over expression might be a good prognostic factor of AML and could be a useful target for immunotherapy.

Keywords: Acute myeloid leukemia; PRAME gene; Prognosis; Real time PCR

\section{Introduction}

Acute myeloid leukemia (AML) is a clonal hematopoietic disorder that may be derived from either a hematopoietic stem cell or a linagespecific progenitor cell. AML is characterized by both; a predominance of immature forms and loss of normal hematopoiesis [1]. The PRAME gene is a cancer/testis (CT) antigen; it was discovered by Ikeda and his colleagues in a melanoma patient in 1997. This gene, which is located in 22q11 of the human chromosome and codes for a protein with 509 amino acids, is a tumor antigen recognized by HLA- 24 and afterward presented to cytotoxic lymphocytes (CTL) [2]. In normal tissues, PRAME gene is expressed in the testis, adrenal gland, ovary and endometrium. Studies abroad suggest that the PRAME gene is highly expressed in leukemic cells. Furthermore, it is known that the overexpression of genes encoding antiapoptotic proteins leads to the survival of leukemic cells via exclusion of apoptosis and its expression levels are correlated with the relapse and remission of leukemia [3]. The mRNA level of PRAME is used as a tumor marker due to its over expression in various malignancies. The PRAME transcript is highly expressed in AML patients and is usually associated with a favorable prognosis [4]. Continuous gene expression monitoring during treatment could determine the effect of chemotherapy and whether leukemic cells are drug resistant, however; local investigations and data on PRAME gene are rare [5]. PRAME gene is a widely expressed leukemia gene that has been the focus of much scientific research recent years.

This gene is not only widely expressed in many hematological malignant diseases, but is also intimately related to the progression and the remission of diseases [6]. The regulation of PRAME gene expression is poorly understood, and thus the molecular basis of its expression in malignancies is largely unknown. It has been suggested that AML1-ETO and BCR-ABL fusion proteins may contribute to the up-regulation of PRAME [7]. PRAME up-regulation was observed in most AML cases with $\mathrm{t}(8 ; 21)$ karyotype and $45 \%$ of AML cases with $\mathrm{t}(15 ; 17)[8]$.

\section{Aim of the work}

To determine the expression of PRAME gene in AML patients and its importance in the prognosis.

\section{Subjects and Methods}

This study was carried out in Clinical Pathology and Medical Oncology Departments, Faculty of Medicine, Zagazig University Hospitals. All patients gave their consent to participate in the study and to allow their biological samples to be analyzed. The study protocol was approved by the Ethical Committee of Faculty of Medicine, 
Zagazig University. The study included 112 subjects. They were classified as following:

\section{Control subjects}

This group included 40 apparently healthy subjects ( 20 males and 20 females). Their ages ranged from 18 to 65 years.

\section{AML subjects}

This group included 72 de novo AML patients (40 males and 32 females). Their ages ranged from 18 to 62 years, they were diagnosed based on standard morphology, cytochemistry and immunophenotyping of the leukemic blasts. All of the patients were treated with anthracyclin and Ara-C as induction chemotherapy schedules; Visinoid was added in M3 cases.

\section{Follow up}

Patients were followed up for 18 months, to assess the response to therapy, relapse if occurred (with routine laboratory investigations and B.M examination), and survival.

\section{Patients were subjected to the following}

Thorough history taking and full clinical examination. Complete Blood Count (CBC), bone marrow aspirate examination, cytochemistry and immunophenotyping using fluorescene labeled mouse monoclonal antibodies to specific myeloid cluster of differentiation (CD) included (CD13,CD33,CD14,CD64 and MPO) and progenitor markers (HLA-DR and CD34) by using Flow cytometry (a FAC-scan, Becton Dickinson, San Jose, California, USA). FACs-acquisition and analysis were performed with FACs Cell Quest software (BD Biosciences) for establishment of the diagnosis of AML [9]. Cytogenetic analysis was done by karyotyping and FISH techniques, and the patients were classified according to their standardized cytogenetic risk subgroups [10].

\section{Detection of PRAME Gene by Real-time RT-PCR}

All members of this study were subjected to relative quantitative expression of PRAME gene in peripheral blood by Real-time RT-PCR technique. This method involved extraction of mRNA, reverse transcription to cDNA then selective amplification by Real-Time PCR System using DNA fluorescent dyes [11].

\section{RNA extraction}

RNA was purified from K-EDTA anti-coagulated peripheral blood samples using the Total RNA Purification Kit (Jena Bioscience: Jena, Germany).

\section{Reverse transcription}

The reverse transcription reaction was performed by SCRIPT Reverse Transcriptase kit (Jena Bioscience: Jena, Germany).

\section{Real time PCR amplification}

The cDNA was amplified with qPCR Green Master (Jena Bioscience: Jena, Germany).

\section{Primers}

The primers were specific for PRAME gene and B actin that obtained from the BLAST program and purchased as lyophilized reagents (Metabion international AG). The forward sequence of PRAME gene primer was 5'-CTGTACTCATTTCCAGAGCCAGA-3'. And the Reverse primer sequence was 5' TATTGAGAGGGTTTCCAAGGGGTT-3'. While B-actin primers sequence was 5'-AGCGAGCATCCCCCAAAGTT-3' for Forward primer and for Reverse primer it was 5'GGGCACGAGGGCTCATCATT-3'.

\section{Steps}

\section{Preparation of the primers}

Final concentration $10 \mu \mathrm{M}$ of the primers were reached by $1: 10$ dilution.

\section{Preparation of the qPCR reaction mix}

The reaction mix with $20 \mu \mathrm{L}$ final volume was prepared by adding $10 \mu \mathrm{L}$ qPCR Green Master, $0.5 \mu \mathrm{L}$ forward primer, $0.5 \mu \mathrm{L}$ reverse primer, $5 \mu \mathrm{L}$ template cDNA and $4 \mu \mathrm{L}$ PCR grade water into real-time PCR wells of the PCR plate.

\section{Recommended cycling conditions}

The following temperature scheme was performed for the amplification using Stratagene Mx3005P qPCR System (Agilent Technologies, Germany) with measuring cycle threshold (CT). PCR reactions were incubated for $10 \mathrm{~min}$ at $95^{\circ} \mathrm{C}$ as initial denaturation and polymerase activation, after which the target was amplified with 40 cycles, for $15 \mathrm{sec}$ at $95^{\circ} \mathrm{C}$ as denaturation and $1 \mathrm{~min}$ at $58^{\circ} \mathrm{C}$ as annealing and extension.

\section{Results interpretation}

The transcription levels of target genes were normalized to those of $\mathrm{B}$-actin which used as reference gene to account for the variability in the amount of cDNA in each sample. CT value for each reaction was obtained and the delta CT was calculated by subtraction of CT values for the reference gene form CT values of the gene of interest. $\left(\Delta C_{T}=C_{T}\right.$ target $-\mathrm{C}_{\mathrm{T}}$ reference), the delta-delta $\mathrm{CT}$ for each patient was calculated by subtraction of the average of control $\Delta \mathrm{CT}$ from the patient $\Delta \mathrm{CT}(\Delta \Delta \mathrm{CT}=\Delta \mathrm{CT}$ sample - Average $\Delta \mathrm{CT}$ control $)$.

The fold change for gene expression in the sample relatively to the control was calculated by the negative value of the delta-delta CT subtraction $(-\Delta \Delta \mathrm{CT})$ becomes the exponent of 2 where $\mathrm{R}=2^{-\Delta \Delta \mathrm{CT}}$ [12]. The expression of target genes was presented as fold change of gene expression relative to control (control considered 1).

\section{Statistical analysis}

Disease free survival (DFS) was calculated from complete remission (CR) to disease relapse and overall survival (OS) was calculated from diagnosis till death or last follow up. Data were checked, entered and analyzed using SPSS version 19 (SPSS Inc., Chicago, IL). Numerical data were expressed as mean and standard deviation; qualitative data were expressed as frequency and percentage. Mann Whitney $U$ test and Kraskall Wallis $\mathrm{H}$ test were used to compare between studied groups, Spearman-rho method was used to test correlation between 
Citation: $\quad$ Ahmad Baraka, Mona E. Hashem1, Shereen EIShorbagy, Mona hassanein, Salah F. Elsayed (2016) Clinical Importance of PRAME

numerical variables and Logrank test was used in survival analysis. $\mathrm{P}$ value $<0.05$ was considered statistically significant.

\section{Results}

In this study, the mean of age \pm SD in AML patients was $46.1 \pm 15.6$, male to female ratio was (1.3:1) and they were categorized according to FAB sub-classification; the most commonly encountered FAB subgroup was M2 (28/72, 38.9\%), followed by M1 (12/72, 16.7\%) and M4 (12/72, 16.7\%). AML (M3) patients were (8/72, 11.1\%) while AML (M5a) was (4/72, 5.6\%) and M5b (8/72, 11.1\%). As regard cytogenetic analysis, they included 35 normal karyotyping AML patients whom were considered as intermediate risk, while 37 patient had abnormal karyotyping comprised 8 patients had $\mathrm{t}(15 ; 17), 8$ patients had $\mathrm{t}(8 ; 21)$, 4 patients had inversion 16 whom were considered good risk, however unfavorable cytogenetics as $t(6 ; 9)$ was found in 8 patients, 4 patients had trisomy 8 and 5 patients had trisomy 11 .

\section{Comparison between patients and controls regarding PRAME gene expression}

PRAME gene was highly expressed in (74\%) of AML patients. A highly significant increase of PRAME gene expression was recorded in patients compared to controls. The gene expression value had a mean \pm $\mathrm{SD}$ of $3.5 \pm 2.1$ fold change in the patients group compared to a mean $\pm \mathrm{SD}$ of $1 \pm 0$ in the control group ( $\mathrm{p}=0.016)$, (Table1), (Figures 1 and 2).

\begin{tabular}{|l|l|l|l|l|}
\hline Parameter & Cases mean \pm SD & $\begin{array}{l}\text { Controls mean } \pm \\
\text { SD }\end{array}$ & T test & p-Value \\
\hline $\begin{array}{l}\text { PRAME gene } \\
\text { quantitation }\end{array}$ & $3.5 \pm 2.1$ & $1 \pm 0$ & 2.548 & $0.016^{*}$ \\
\hline
\end{tabular}

Table 1: Comparison between Patients and control regarding PRAME gene expression.

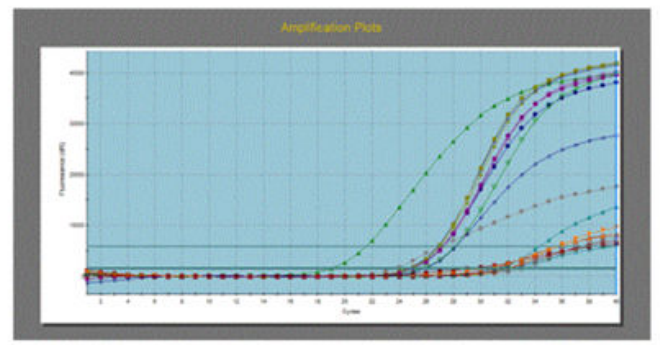

Amplification Plots of PRAME GENE

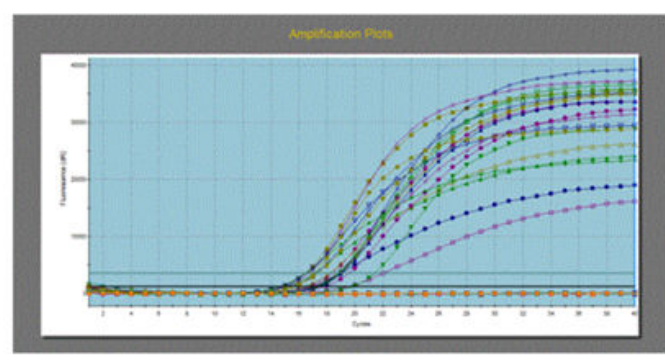

Amplification Plots of Bactin

Figure 1: Comparison between cases and control regarding PRAME gene expression.

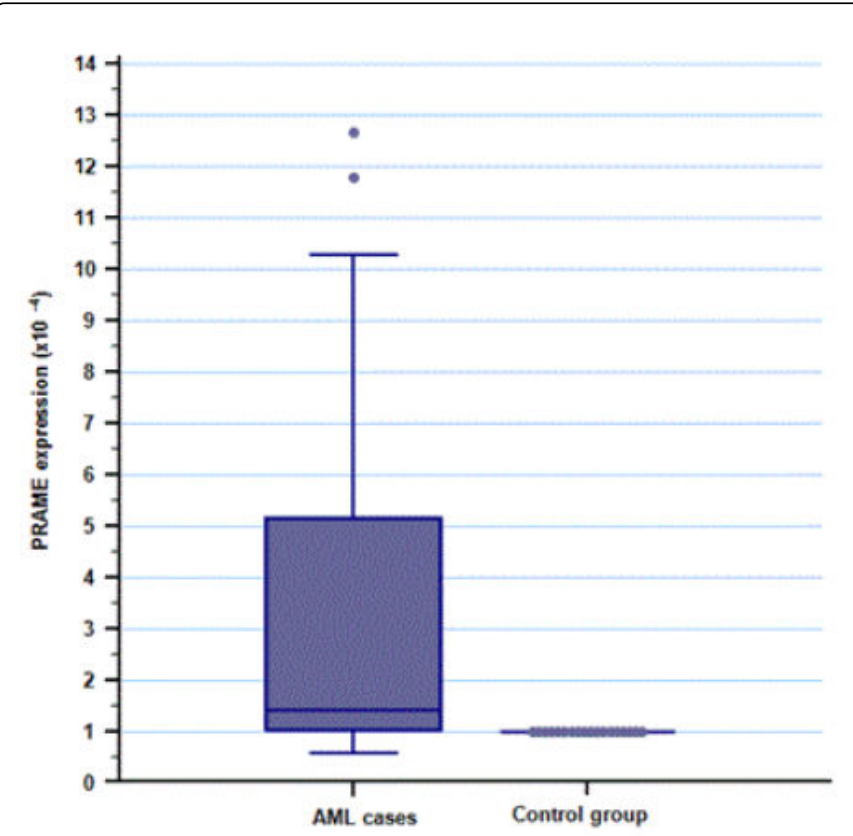

Figure 2: Box-plot shows comparison between AML cases and control group as regard PRAME gene expression $\left(\times 10^{-4}\right)$.

As regard PRAME gene expression, there was no statistically significant relation with age, sex, hematological parameters $(\mathrm{CBC}$ and B.M blast \%) or cytogenetic abnormalities.

However, there was a statistical significance difference between FAB subgroups, as a significant high gene expression was found in M3 more 
Citation: $\quad$ Ahmad Baraka, Mona E. Hashem1, Shereen ElShorbagy, Mona hassanein, Salah F. Elsayed (2016) Clinical Importance of PRAME

Page 4 of 7

than other FAB subtypes followed by M2, then M5a, M4, M1 and lastly M5b showed the lowest gene expression among the FAB subtypes $(\mathrm{P}=0.01)$, (Table 2).

\begin{tabular}{|c|c|c|c|}
\hline \multirow[t]{2}{*}{ Variables } & \multicolumn{2}{|c|}{ PRAME gene expression } & \multirow{2}{*}{$\begin{array}{l}\text { p-Value } \\
\text { (Sig.) }\end{array}$} \\
\hline & Mean \pm SD & Median (Range) & \\
\hline \multicolumn{4}{|l|}{ Age } \\
\hline$\leq 45$ years & $321.35 \pm 0.38$ & $1.34(0.85-1.95)$ & 0.286 \\
\hline$>45$ years & $405.34 \pm 4.96$ & $3.21(0.59-12.66)$ & (NS) \\
\hline \multicolumn{4}{|l|}{ Sex } \\
\hline Male & $403.73 \pm 4.29$ & $1.41(1.02-12.66)$ & 0.424 \\
\hline Female & $323.37 \pm 4.25$ & $1.32(0.59-11.80)$ & (NS) \\
\hline \multicolumn{4}{|l|}{ Cytogenetic risk } \\
\hline Intermediate risk(normal) & $352.15 \pm 3.06$ & $1.12(0.59-10.28)$ & \\
\hline Good risk & $204.99 \pm 4.76$ & $1.70(0.85-12.66)$ & 0.2 \\
\hline Poor risk & $171.6 \pm 3.4$ & $1.2(0.5-6.2)$ & (NS) \\
\hline \multicolumn{4}{|l|}{ FAB } \\
\hline M1 & $121.10 \pm 0.07$ & $1.12(1.02-1.17)$ & \\
\hline M2 & $284.12 \pm 3.86$ & $1.70(0.8-10.28)$ & \\
\hline M3 & $812.23 \pm 0.60$ & $12.23(11.80-12.66)$ & 0.01 \\
\hline M4 & $121.37 \pm 0.31$ & $1.37(1.15-1.60)$ & $(\mathrm{S})$ \\
\hline M5a & $41.95 \pm 0.9$ & $2.1(0.53-2.8)$ & \\
\hline M5b & $80.97 \pm 0.35$ & $1.04(0.59-1.29)$ & \\
\hline
\end{tabular}

Table 2: Relation between PRAME expression and demographic, cytogenetic risk and FAB classification in AML patients.

\section{PRAME gene expression and response to induction chemotherapy}

Complete remission (CR) after induction chemotherapy achieved when $\mathrm{BM}$ shows blast cells $<5 \%$ with normal maturation of all cell lines, absence of blast cells from PB, clearance of disease associated CG abnormality and clear of any previously existing extramedullary disease, in our study; the (CR) was achieved in $40 / 72$ patients $(55.5 \%)$ (responder), compared to $32 / 72$ (44.4\%) who failed to achieve CR (non-responder) after the induction chemotherapy. As regard PRAME gene expression, there was a significant high level of expression in responders compared to non-responders $(\mathrm{p}=0.026)$, (Table 3 ).

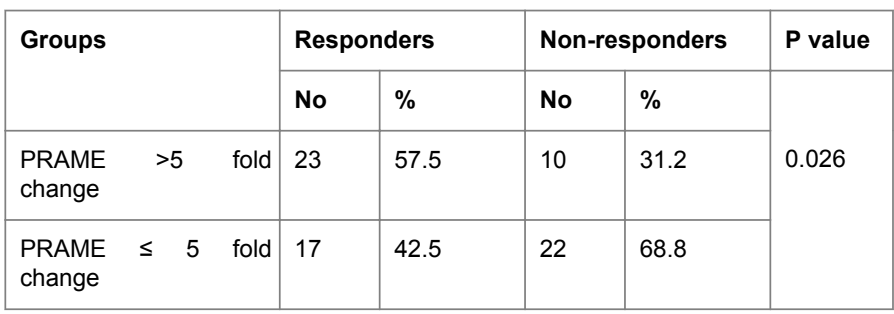

Table 3: Comparison between responders and non-responders as regard PRAME gene expression.

\section{PRAME gene expression and survival analysis}

During the follow up period, leukemia relapse was observed in $12 / 40$ after complete remission and 26 patients (36\%) died.

\section{Disease free survival (DFS)}

12 months and 18 months DFS for all patients were $85.0 \%$ and $70.0 \%$, respectively. In studying relation between PRAME gene expression and DFS, It was found that there was a statistical significance correlation between PRAME gene expression and DFS as with PRAME $\leq 5$ fold change, 12 months and 18 months DFS were $76 \%$ and $53 \%$, respectively, while with gene expression $>5$ fold change, 12 months and 18 months DFS were $91 \%$ and $82 \%$ respectively $(\mathrm{p}=0.04)$ (Table4) \& (Figure3).

\begin{tabular}{|c|c|c|c|c|}
\hline DFS and OS & PRAME $\leq 5$ fold change & PRAME >5fold change & Test $^{*}$ & p-Value (Sig.) \\
\hline Median DFS & 14 months & NR & \multirow[t]{3}{*}{3.9} & \multirow[t]{3}{*}{$0.04(\mathrm{~S})$} \\
\hline 12months DFS & $76 \%$ & $91 \%$ & & \\
\hline 18months DFS & $53 \%$ & $82 \%$ & & \\
\hline Median OS & 10 months & NR & \multirow[t]{3}{*}{15.6} & \multirow[t]{3}{*}{$<0.001(\mathrm{HS})$} \\
\hline 12months OS & $46 \%$ & $94 \%$ & & \\
\hline 18months OS & $38 \%$ & $91.40 \%$ & & \\
\hline
\end{tabular}

Table 4: Relation between disease free survival (DFS), overall survival (OS) and PRAME gene expression. 
Page 5 of 7

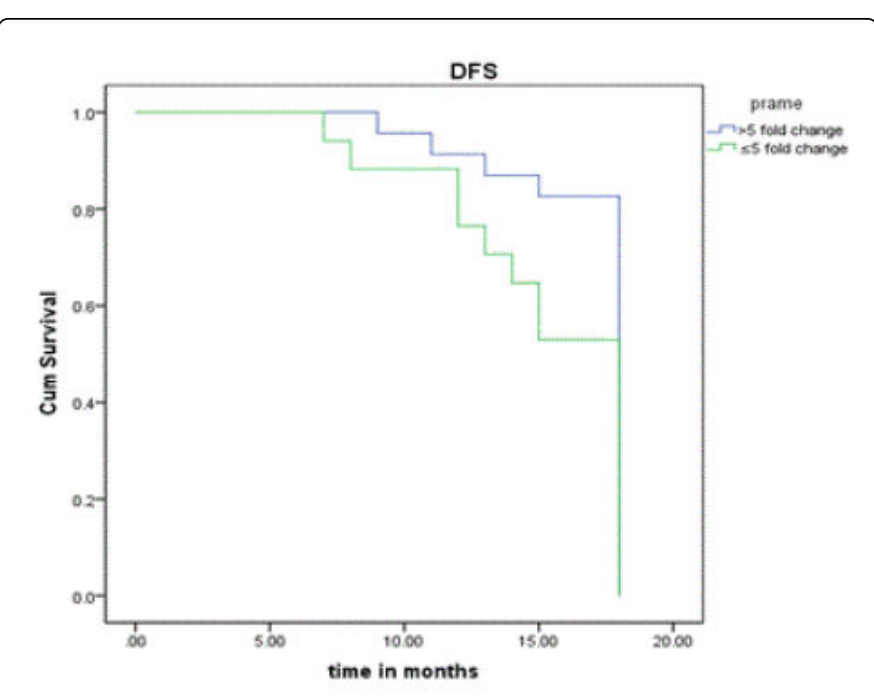

Figure 3: Kaplan Meier plot of DFS in AML patients according to PRAME gene expression.

\section{Overall survival (OS)}

The median overall survival of all patient with AML was 15 months, with $23 \%$ for 18 month overall survival, while 12 months overall survival was $62.8 \%$. In studying relation between PRAME gene expression and OS, It was found that; with PRAME $\leq 5$ fold change, 12 months and 18 months OS were $46 \%$ and $38 \%$, respectively while with gene expression $>5$ fold change, 12 months and 18 months OS were $94 \%$ and $91.4 \%$, respectively so, there was a statistical significance correlation between PRAME gene expression and overall survival value $(\mathrm{p}<0.001)$, (Figure 4$)$.

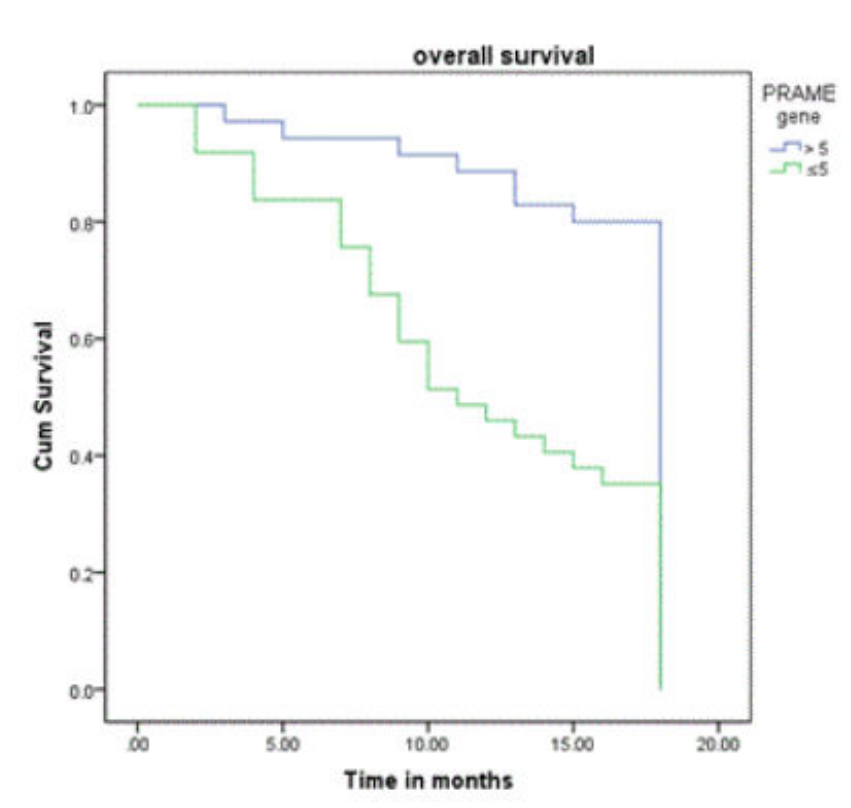

Figure 4: Kaplan Meier plot of overall survival in AML patients according to PRAME gene expression.

\section{Discussion}

Acute myeloid leukemia (AML) is a hematopoietic malignant disease rising from neoplastic transformation of myeloid stem cells. This causes the alteration of differentiation and proliferation systems, resulting in the accumulation of non-functional myeloid cells termed myeloblasts in bone marrow and peripheral blood [13]. PRAME gene is a tumor antigen, but its role has not been fully elucidated, so far; it has been found to be expressed in a variety of hematological malignancies [14].

Our study aimed to determine the expression of PRAME gene in AML patients and its prognostic value. PRAME gene expression was detected at low level in peripheral blood samples obtained from normal healthy donors, while it was highly expressed in fifty three AML patients (74\%). Ding et al., [6] found that, PRAME gene was expressed in $40.7 \%$ of cases, while Steinbach et al., [15] recorded that it was expressed in $62 \%$ of cases. These variations may have been attributed to differences in the patients races examined. Like the findings of other studies, low PRAME gene expression was found in the healthy volunteers. This tumor restricted expression would be favorable for future immunotherapy, because of absence of danger to induce autoimmune reactions by vaccination of patients with these TAAs/LAAs [16] (Table 5).

\begin{tabular}{|l|l|l|}
\hline \multirow{2}{*}{ Lab parameters } & \multicolumn{2}{|l|}{ PRAME gene expression } \\
\cline { 2 - 3 } & $\mathbf{R}$ & $\mathbf{p}$-Value \\
\hline TLC $(\times 109 / \mathrm{L})$ & 0.131 & 0.605 \\
\hline $\mathrm{Hb}(\mathrm{g} / \mathrm{dl})$ & 0.144 & 0.569 \\
\hline Platelets (( $109 / \mathrm{L})$ & -0.27 & 0.278 \\
\hline B.M Blast \% & -0.086 & 0.734 \\
\hline
\end{tabular}

Table 5: Correlation between hematological parameters and PRAME gene expression in the patients tab.

In this study, acute promyelocytic leukemia patients (M3) had the highest gene expression rate followed by M2, and lastly M5b, similar to the results of Paydas et al., and Van Baren, in addition to the study done by Spanaki and colleagues which denoted that PRAME mRNA expression level was higher for AML (M3) patients with $t(15 ; 17) "$ PML-RARa" [17]. In a study carried by Zhu et al., they reported that, among the FAB subtypes, those with M1, M2, M3 and M4 had significantly higher level of PRAME transcripts than controls, however, those with M5 had similar level of PRAME transcripts as controls, also among cases with AML-M2, those with $\mathrm{t}(8 ; 21)$ had significantly higher level of PRAME transcripts than those without $t(8 ; 21)$ [18].

No significant relation of PRAME gene expression with cytogenetic risk groups was reported in our patients $(\mathrm{p}=0.2)$. Several studies have demonstrated that, PRAME gene is correlated with chromosomes translocation. However, the functional and laboratory association between PRAME gene and variant chromosomal translocations need further investigations [8].

In this study, no significant correlation of PRAME gene expression with age, sex, TLC, $\mathrm{Hb}$ level, platelets count, or blast percentage in BM was observed, this was similar to Paydas' study. On the contrary, in a pediatric study done by Steinbach et al., [15] they found that PRAME gene expression was negatively correlated to white blood cell count at diagnosis. Spanaki and colleagues [13], denoted that in spite of no 
Page 6 of 7

correlation between PRAME gene expression and clinical parameters including CBC parameters, PRAME gene could serve as a marker for monitoring MRD in acute leukemia.

As regards disease outcome, many studies discussed the role of PRAME gene expression in outcome and prognosis among AML cases. Theories were not all in agreement and may contradict [14]. Our patients were followed up for 18 months (range 2 to 18), 55.5\% of cases achieved CR with intensive chemotherapy, $30 \%$ of CR cases were relapsed, and $36.0 \%$ of cases died. PRAME gene expression was significantly high in responders compared to non-responders $(\mathrm{p}=0.026)$. As regards DFS studies, there was a statistical significant association between high PRAME gene expression and better DFS $(\mathrm{p}=0.020)$. In the current study the median overall survival of all patients was 15 months with a significant association between high PRAME gene expression and better overall survival, $(\mathrm{p}<0.001)$. while Paydas et al., didn't find any correlation between PRAME expression and progression-free or overall survival, however, this may be explained by different protocols of therapy or variance in other prognostic factors. On the contrary, our results agree with Greiner et al., [16] who found a significant correlation between high m-RNA levels of PRAME and longer overall survival. Also in a pediatric study, Steinbach et al., had found that the rates of overall and disease-free survival in patients with high PRAME expression were higher than in patients with no or low expression, suggesting that the expression of the PRAME gene could be used as an indicator of good prognosis for risk adapted therapy [15].

Another study of Tajeddine and colleagues [19] explained that PRAME induces Caspase-Independent Cell Death in vitro and reduces tumorgenicity in vivo. PRAME expression in many malignancies is an indicator of aggressive disease and poor prognosis but in AML, it is associated with favorable chromosomal aberrations and a relatively good prognosis.

Greiner and colleagues [16] observed a trend towards longer OS among cases with higher PRAME gene and suggested that the expression of distinct TAAs/LAAs as PRAME on leukemic blasts might enable the immune system to better eradicate MRD following intensive chemotherapy, there by leading to decreased relapse rates and longer OS times.

Epping and colleagues [20] showed that PRAME mRNA expression in adult de novo AML cases is not associated with poor prognosis (outcome). Epping showed that PRAME is a repressor of retinoic acid signaling. Retinoic acid induces proliferation but arrest differentiation and apoptosis. PRAME binds to the retinoic acid receptor in the presence of retinoic acid preventing ligand-induced receptor activation and target gene transcription. As a result, PRAME could confer growth or survival advantages to cancer cells including AML cells by antagonizing retinoic acid receptor signaling. In contrary to all previous studies, Goellner and colleagues in their 2006 study stated that patients with PRAME up regulation might be more resistant to chemotherapy [21].

\section{Conclusion}

PRAME gene overexpression might be a good prognostic factor of AML and could be a useful target for immunotherapy.

\section{Recommendation}

Employ Real Time PCR to quantitatively assess level of PRAME gene expression at diagnosis, during the course of therapy and after CR. AML patients should be monitored using Real Time PCR to efficiently evaluate the serial changes of the gene expression among cases at diagnosis, during chemotherapy and during the follow up period of CR so, we can rely on a useful tool for detecting MRD and to predict subsequent relapse especially in patients without known genetic markers.

\section{Conflict of Interest}

The authors indicated no potential conflict of interest.

\section{References}

1. Vardiman JW, Thiele J, Arber DA, Brunning RD, Borowitz MJ, et al. (2009) The 2008 revision of the World Health Organization (WHO) classification of myeloid neoplasms and acute leukemia: rationale and important changes. Blood 114: 937-951.

2. Ikeda H, Lethe B, Lehmann F, Van Baren N, Baurain JF, et al. (1997) Characterization of an antigen that is recognized on a melanoma showing partial HLA loss by CTL expressing an NK inhibitory receptor. Immunity 6: 199-208.

3. Paydas S, Tanriverdi K, Yavuz S, Disel U, Baslamisli F, et al. (2005) PRAME mRNA Levels in Cases With Acute Leukemia: Clinical Importance and Future Prospects. Am J Hematol 79: 257-261.

4. Amira M Khorshed, Ghada I Mossallam et al. (2012). Expression of PRAME gene in Egyptian adult acute myeloid leukaemia and its correlation with clinical response. Life Sci J 9: 1117-1121.

5. Matsushita M, Ikeda $H$, Kizaid M, Okamoto $S$, Ogasawara $M$, et al. (2001) Quantitative monitoring of the PRAME gene for the detection of minimal residual disease in leukemia. Br J haematol 112: 916-926.

6. Ding K, Wang KM, Rong Fu, Er-baoRuan, , et al. (2012) PRAME Gene Expression in Acute Leukemia and Its Clinical, cancer biol med 9: 73-76.

7. Watari K, Tojo A, Nagamura-Inoue T, Nagamura F, Takeshita A, et al. (2000) Identification of a melanoma antigen, PRAME, as a BCR/ABLinducible gene. FEBS Lett 466: 367-371.

8. Qin YZ, Li JL, Zhu HH, Li LD, Chang Y, et al. (2009). PRAME mRNA expression in newly diagnosed acute myeloid leukemia patients and its application to monitoring minimal residual disease. Zhonghua Xue Ye Xue Za Zhi 29: 441- 451.

9. Wood BL, Arroz M, Barnett D, DiGiuseppe J, Greig B, et al. (2007) 2006 Bethesda International Consensus recommendations on immunophenotypic analysis of hematolymphoid neoplasia by flow cytometry: optimal reagents and reporting for the flow cytometric diagnosis of hematopoietic neoplasia . Cytometry B Clin Cytom 72 Suppl1: S14 - S22

10. Döhner H, Amadori S, Appelbaum FR, Büchner T, Burnett KA, et al. (2010) Diagnosis and management of acute myeloid leukemia in adults: recommendations from an international expert panel, on behalf of the European LeukemiaNet. Blood 115: 453-474.

11. Freeman WM, Walker SJ and Vrana KE (1999) "Quantitative RT-PCR: pitfalls and potential". Biotechniques 26: 112-122, 124-125.

12. Livak KJ \& Schmittgen TD (2001) Analysis of relative gene expression data using real-time quantitative PCR and the 2(-Delta Delta C (T)) Method. Methods 25: 402-408.

13. Sarah Parisi \& Antonio Curti (2012) Targeting the Minimal Residual Disease in Acute Myeloid Leukemia:The Role of Adoptive Immunotherapy with Natural Killer Cells and Antigen-Specific Vaccination. ISBN: 978-953-51-017-1.

14. Spanaki A, Perdikogianni C, Linardakis E , Kalmanti M (2007) Quantitative assessment of PRAME expression in diagnosis of childhood acuteleukemia. Leuk Res 31: 639-642. 
Citation: Ahmad Baraka, Mona E. Hashem1, Shereen ElShorbagy, Mona hassanein, Salah F. Elsayed (2016) Clinical Importance of PRAME Gene Expression in Acute Myeloid Leukemia. J Blood Disord Transfus 2016, 7: 373 7: 373. doi:10.4172/2155-9864.1000373

Page 7 of 7

15. Steinbach D, Hermann J, Viehmann S, Zintl F, Gruhn B (2007) Clinica implications of PRAME gene expression in childhood acute myeloid leukemia. Cancer Genet Cytogenet 133: 118-123.

16. Greiner J, Schmitt M, Li L, Giannopoulos K, Katrin B, et al. (2006) Expression of tumor associated antigens in acute myeloid leukemia: implications for specific immunotherapeutic approaches. Blood journal. Blood 108: 4109-4117.

17. Van Baren N, Chambost H, Ferrant A, Michaux, Ikeda, et al. (1998) PRAME, a gene encoding an antigen recognized on a human melanoma by cytolytic T cells, isexpressed in acute leukaemia cells. Br J Haematol 102:1376-1379.

18. Zhu Z, Qian J, Lin J, Yao D, Qian Z, et al. (2010). Quantification of the PRAME transcripts in patients with acute myeloid leukemia. Zhonghua Yi Xue Yi Chuan Xue Za Zhi 27: 149-152.
19. Tajeddine N, Louis M, Vermylen C, Gala JL, Tombal B, et al. (2008) Tumor associated antigen PRAME is a marker of favorable prognosis in childhood acute myeloid leukemia patients and modifies the expression of S100A4, Hsp 27, p21, IL-8 and IGFBP-2 in vitro and in vivo Leuk Lymphoma 49: 1123-1131.

20. Epping MT, Wang L, Plumb JA, Lieb M, Gronemeyer H, et al. (2007) A functional genetic screen identifies retinoic acid signaling as a target of histone deacetylase inhibitors. Proc Natl Acad Sci USA 104: 17777-17782.

21. Goellner S, Steinbach D, Schenk T, Gruhn B, Zintl F, et al. (2006) Childhood acute myelogenous leukaemia: association between PRAME, apoptosis- and MDR-related gene expression. Eur J Cancer 42: 2807-2814. 